\title{
Interference in the angular distribution of photoelectrons in superimposed XUV and optical laser fields
}

\author{
S. Düsterer \\ Deutsches Elektronen-Synchrotron (DESY), Notkestrasse 85, D-22603 Hamburg, \\ Germany
}

\section{Rading, P. Johnsson}

Lund University, P.O. Box 118, SE-221 00 Lund, Sweden

\author{
A. Rouzée, A. Hundertmark, M. J. J. Vrakking \\ Max Born Institute, Max Born Strasse 2A, 12489, Berlin, Germany
}

\section{P. Radcliffe, M. Meyer}

European XFEL GmbH, Albert-Einstein-Ring 19, D-22761 Hamburg, Germany

\author{
A. K. Kazansky \\ Departamento de Fisica de Materiales, University of the Basque Country UPV/EHU, \\ E-20018 San Sebastian/Donostia, Spain \\ Donostia International Physics Center (DIPC), E-20018 San Sebastian/Donostia, \\ Basque Country, Spain \\ IKERBASQUE, Basque Foundation for Science, E-48011 Bilbao, Spain
}

\author{
N. M. Kabachnik \\ Skobeltsyn Institute of Nuclear Physics, Lomonosov Moscow State University, \\ Moscow 119991, Russia \\ European XFEL GmbH, Albert-Einstein-Ring 19, D-22761 Hamburg, Germany \\ E-mail: stefan.duesterer@desy.de
}

\begin{abstract}
.
The angular distribution of photoelectrons ejected during the ionization of $\mathrm{Ne}$ atoms by extreme ultraviolet (XUV) free-electron laser radiation in the presence of an intense near infrared (NIR) dressing field were investigated experimentally and theoretically. The highly nonlinear process with absorption and emission of more than 10 NIR photons results in the formation of numerous sidebands. The intensity of the sidebands varies strongly with the emission angle and the angular distribution pattern reveals clear signatures of interferences between the different angular momenta for the outgoing electron in the multi-photon process. As a specific feature, the central photoelectron line is characterized at highest NIR fields by an angular distribution,
\end{abstract}


which is peaked perpendicularly to both the XUV and NIR polarization direction. The experimental results are reproduced by a theoretical model based on the strong field approximation.

PACS numbers: $32.80 . \mathrm{Wr}, 32.80 . \mathrm{Aa}, 32.80 . \mathrm{Fb}$

Submitted to: J. Phys. B: At. Mol. Phys.

Free-Electron Lasers (FELs) operating in the extreme ultraviolet (XUV) and X-ray regime $[1,2,3]$, featuring unrivaled high photon fluxes, have rapidly become important tools for investigating nature at extreme conditions $[4,5,6]$. These sources allow to investigate atoms in strong fields with high photon energies. Combining XUV FELs with intense optical laser fields opens up a wide range of laser-assisted photoionization experiments as the control of the relaxation of resonant core hole states in either resonant or normal Auger decay due to the influence of the optical field [7] or to study timeresolved the relaxation pathways in Auger cascades [8].

Photoelectrons emitted during many cycles of a strong optical or NIR field experience the quantum nature of the dressing field and can be shifted in energy by an integer number of optical quanta, $\pm \hbar \omega_{N I R}$. The central photo line is split in a multitude of lines - the so-called sidebands. In the quantum mechanical picture the appearance of sidebands is the result of interference between electron waves emitted at different periods of the optical laser pulse [9]. The corresponding experiments provide in this way a unique test ground for studying fundamental multi-photon processes and for testing the validity of theoretical approaches aiming to describe these nonlinear phenomena. Sidebands were extensively investigated using high order harmonic generation (HHG) sources [10] and they were proven to be an ideal tool to characterize the HHG emission, on both femtosecond and attosecond time scales[11, 12]. Later the approach was transferred to FEL experiments, for which, besides the characterization of the source, the non-linear processes induced by the optical laser could be investigated more clearly, but so far relying mainly on angle integrated measurements $[13,14]$. More information on the photoionization process is obtained by angle-resolved experiments, which have been so far only performed at either low optical intensities with HHG sources (only one sideband) $[16,15]$ or for low energetic electrons in above-threshold ionization (ATI) $[17,18,19,20,21,22]$. In the latter case the angular distribution of the ejected electrons were reported for the one-color multi-photon case.

Theoretical studies of the angularly resolved photoelectron spectra in XUV-optical two-color experiments at high optical intensities show as well quantum mechanical interference structures, when more and more photons are involved. This results in oscillations in the angular distribution of photo and Auger electrons. The sideband amplitudes for a given emission angle exhibit variations as a function of the photoelectron kinetic energy, the so-called gross structure, due to the interference of electrons emitted within one optical cycle $[9,23,24,25]$. These calculations were 
performed for ultra-short XUV or X-ray pulses with a duration comparable to the oscillation period of optical light, i.e. a few fs. Recently, the above-mentioned gross structure was experimentally observed in the laser-assisted Auger decay of Ne atoms [26] using X-ray FEL pulses of such short nature. In these experiments the life time of the Auger process was on the time scale of the oscillation period of the optical laser.

In the present paper, we report on the angle-resolved observation of the sideband formation in XUV direct photoionization of Ne atoms at high NIR intensities yielding up to ten sidebands. Here, the XUV pulse duration is much longer ( 30 optical cycles) than discussed in the previous calculations. However, we still observed strong oscillations in the angular distribution as well as in the sideband amplitudes for given angles. In particular there is a distinct asymmetry comparing the features occurring at higher energetic side of the initial photoionization line and the corresponding lower energetic one. The oscillatory structures are altered by changing the peak field of the NIR dressing laser, but the interference is still present when the experimental data is integrated over many different intensities. The experimentally observed angular variations can be interpreted as interferences between many outgoing partial waves in the multi-photon ionization. The observations are reproduced by our theoretical model.

The experiments were performed at beam line BL2 of the Free-electron LASer in Hamburg (FLASH)[1]. FLASH was operated at a wavelength of $13.7 \mathrm{~nm}(90.5 \mathrm{eV})$ and a bandwidth of less than $1 \%(<0.9 \mathrm{eV})$. The pulse duration was $\sim 80$ fs and the pulse energy 30-40 $\mu \mathrm{J}$. For the two-color experiments we also used the optical laser system at FLASH [27]. The laser delivered also 80 fs pulses at a central wavelength of $800 \mathrm{~nm}$; peak intensities in the order of $10^{13} \mathrm{~W} / \mathrm{cm}^{2}$ could be reached in the focus. Both XUV and NIR pulses were linearly polarized in the horizontal plane. The optical laser and the FEL pulses are synchronized electronically to each other up to a remaining pulse to pulse jitter of $\sim 0.5 \mathrm{ps}(\mathrm{FWHM})[27]$. This jitter leads to significant shot-to-shot variations of the effective optical intensity seen by the ionized electrons. Thus recording electron spectra for each single FEL pulse and subsequent resorting of the data as described below are essential for the success of the experiment.

In the experimental chamber, the FEL-beam and the optical laser are combined using a $45^{\circ}$ holey mirror and crossed with a pulsed gas jet. The resulting photoelectrons are detected with a Velocity Map Imaging Spectrometer (VMIS) [28]. The VMIS is ideally suited to perform momentum imaging of the charged particles resulting from ionization by the high flux FEL as it allows for very high count rates $\left(10^{4}-10^{5}\right.$ events/shot). The image detected by the VMIS is a $2 \mathrm{D}$ projection of the $3 \mathrm{D}$ momentum photoelectron distribution in the interaction region, which can be retrieved by an inverse Abel transform [29]. In order to achieve the spatial and temporal overlap between the FEL pulses and the optical pulses, the capabilities of the VMIS setup were used [30].

Neon was used as target gas and the main ionization channel for the $90.5 \mathrm{eV}$ FEL pulses was Ne $2 \mathrm{p}^{6} \rightarrow \mathrm{Ne}^{+} 2 \mathrm{p}^{5}+\mathrm{e}^{-}(68.9 \mathrm{eV})$. The acquired data set consists of 10.000 single-shot images. To be able to study in more detail how the angular distribution changes with the NIR intensity seen by the photoelectrons, the single-shot images were 
sorted into ten bins depending on the amount of sideband signal, which increases with increasing intensity, compared to the total signal. The effective NIR intensity for every bin was retrieved from the ponderomotive energy shift of the photoelectron lines in respect to the line position without NIR field [31]. Through an FFT-analysis of the photolines, subpixel resolution could be achieved and shifts in the order of $0.2 \mathrm{eV}$ were found. The sorting resulted in about equidistant intensity windows of $0.8 \times 10^{12} \mathrm{~W} / \mathrm{cm}^{2}$ for each bin. The sample image shown in Fig. 1 displays the measured raw data of the VMIS with FEL only and FEL in combination with the strong NIR field (averaged and intensity binned). The sidebands are separated by only $\sim 3$ pixels, thus demonstrating the resolution capabilities of the VMIS. The inverted angularly resolved photoelectron spectra of Ne are shown in Fig. 2 (upper panel) in the presence of a $6 \times 10^{12} \mathrm{~W} / \mathrm{cm}^{2} \mathrm{NIR}$ field.

The experimental spectra bring out several interesting features (Fig. 2, upper panel). First, for each particular angle the intensity of the sidebands clearly shows an oscillatory behavior (gross structure). Furthermore, the low order sidebands and the central line show strong modulations as a function of the emission angle. Higher order sidebands show less modulations and the angular distribution is peaked towards $0^{\circ}$. Thus, the highly accelerated and decelerated electrons are predominantly emitted along the polarization axis of the optical laser. Finally, the modulations are different for the sidebands of the same order on the high and low energy side of the central peak.

A counter-intuitive result has been obtained for the angular distribution when the intensity of the optical laser is increased. This is illustrated in Fig. 3 (upper panel) where the angular distribution of the central emission line is displayed. The angular distribution at low NIR fields resembles the well-known dipole-type distribution of the $\mathrm{Ne} 2 \mathrm{p}^{6} \rightarrow \mathrm{Ne}^{+} 2 \mathrm{p}^{5}+\mathrm{e}^{-}$ionization, which has an emission maximum at zero and $180^{\circ}$ angles corresponding to the emission along the polarization direction of the FEL. In the presence of a stronger optical dressing field, the angular distribution changes dramatically and is characterized by several maxima. In particular, we obtain a distinctly stronger relative contribution to the emission of electrons perpendicular to the polarization of the ionizing radiation until, at the highest NIR intensity, the emission is almost entirely directed away from the polarization of FEL and optical laser. This is in contrast to the known results for the angular distributions of photoelectrons in the one-color multiphoton ionization where the photoelectrons are concentrated along the polarization vector of the laser field (see e.g. [32]).

To interpret the experimental results, simulations have been performed using a theoretical approach based on the strong-field approximation, which is described in detail in Ref. [9]. Namely, it is supposed that the ionization by the XUV pulse occurs in an "undressed" free atom, but the emitted photoelectron, propagating from the atom to the detector, interacts with the strong IR field which changes electron energy and scattering angle. In this approximation the photoelectron is described by the Volkov wave function [33] which contains all partial waves with corresponding fieldmodified phases. This approach works surprisingly well when the photoelectron energy is 


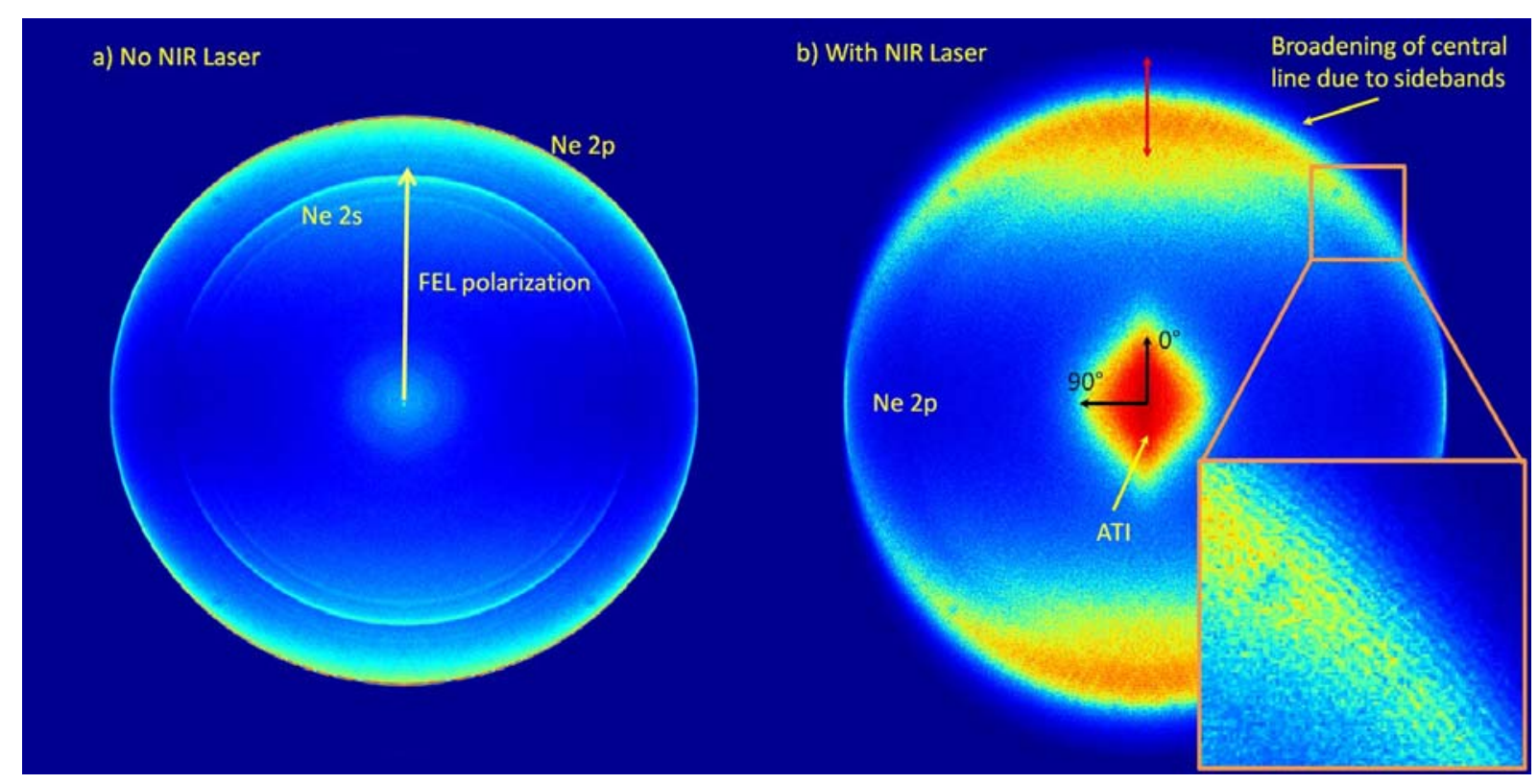

Figure 1. (color online). Experimentally recorded VMIS data for the Ne photoionization at 90.5 eV FEL photon energy is shown for the case with only FEL (a) and FEL in the presence of an NIR (800 nm) intensity of $\sim 3 \times 10^{12} \mathrm{~W} / \mathrm{cm}^{2}$ (b). Shown is the angular dependence of the emitted photoelectrons (the polarization direction of FEL and NIR laser is vertical). In (a) the $2 \mathrm{p}$ as well as the $2 \mathrm{~s}$ (inner ring) are clearly visible (the radius represents the electron momentum). In (b) the NIR laser broadens the central line substantially due to sideband formation. Thus $2 \mathrm{p}$ and $2 \mathrm{~s}$ contributions are not clearly visible anymore. The broadening decreases for larger angles in respect to the polarization till finally at $90^{\circ}$ there is almost no effect of the NIR laser on the photoelectrons. The red double arrow indicates the energy range shown in Fig.2. The low kinetic energy feature in the center results from NIR generated ATI electrons in the rest gas. The data is binned according to the sideband amplitude (in b) and averaged over several hundred single shot images. The enlarged view of the data (inset) shows that successive sidebands are separated by only $\sim 3$ pixels (corresponding to a separation of $1.5 \mathrm{eV}$ ), thus demonstrating the excellent resolution capabilities of the VMIS.

sufficiently large (several tens of eV). Its comparison with much more elaborate method of calculation based on numerical solving the time-dependent Schrödinger equation shows very good agreement [34]. In the present simulation based on this approach it is assumed that both the XUV and NIR pulses have fixed amplitudes and shapes. To simulate the temporal jitter of the FEL pulses, the relative position of the envelope maxima (relative delay) of the two pulses has been varied from zero (maximum overlap) to $\pm 200 \mathrm{fs}$ (practically no overlap). Naturally, this is a rather rough approximation since the XUV pulse has a very complicated temporal shape which is different from shot to shot. Therefore, one can only expect a qualitative agreement of the simulation with experiment. 

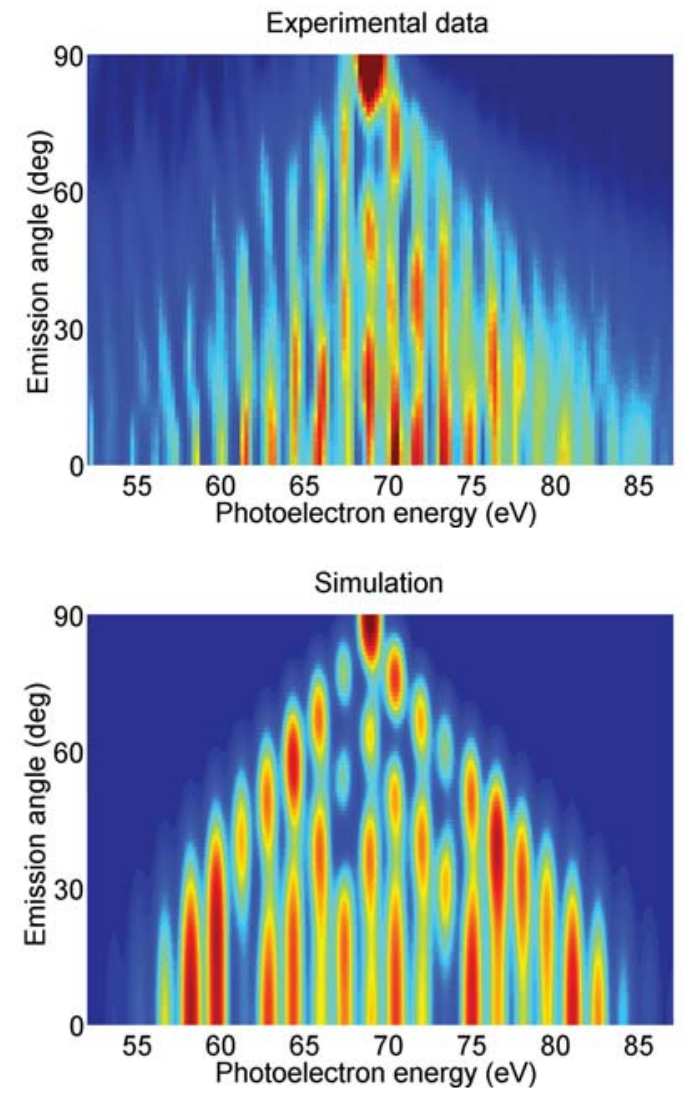

Figure 2. (color online). Upper panel: Photoelectron angular distribution spectra retrieved from an Abel inversion of the measured 2D projection of the electron momentum distribution for the $\mathrm{Ne} 2 \mathrm{p}^{6} \rightarrow \mathrm{Ne}^{+} 2 \mathrm{p}^{5}+\mathrm{e}^{-}$photoionization at $90.5 \mathrm{eV}$ FEL photon energy in the presence of an NIR $(800 \mathrm{~nm})$ intensity of $5-6 \times 10^{12} \mathrm{~W} / \mathrm{cm}^{2}$. Shown are the inverted VMIS data. The polarization angle is defined in respect to the XUV and the NIR polarization. Lower panel: Calculated spectra for the same parameters as used in the experiment

In the calculations presented here (Fig. 2, lower panel) the XUV and NIR pulse have the same pulse duration as in the experiment ( $80 \mathrm{fs}$ FWHM). The NIR field has a peak intensity of $6 \times 10^{12} \mathrm{~W} / \mathrm{cm}^{2}$. The necessary dipole matrix elements and phases have been calculated within the Hartree-Slater approximation [35, 36], which provides the angular distribution parameter for one-photon absorption and has previously showed good agreement with experiments. The calculated spectra for each particular relative delay of the two pulses have been associated with a certain effective NIR intensity by extracting the ponderomotive shift in the same way as for the experimental spectra.

The theoretical double differential cross section is shown in Fig. 2 (lower panel) for the maximum overlap of the two pulses. The comparison with the experimental spectra in Fig. 2 (upper panel) shows quite satisfactory agreement. In particular the simulation shows as well a different oscillatory structure for the sidebands of the same order on the high and low energetic side. This asymmetry between low-energy and high-energy 

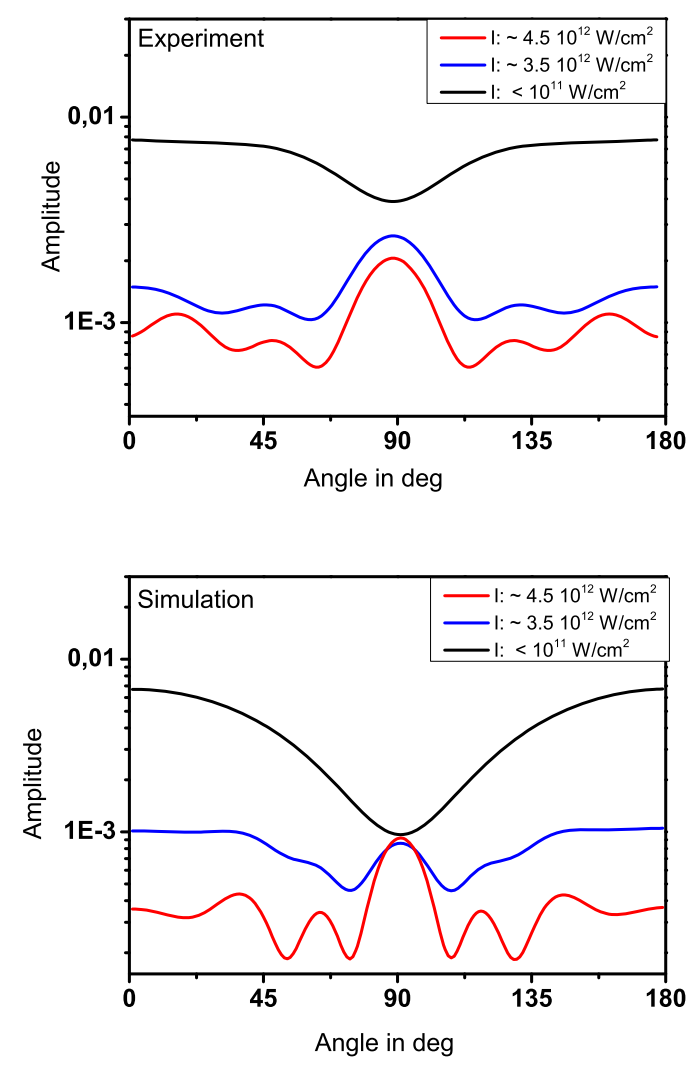

Figure 3. (color online). Angular dependence of the central line at a kinetic energy of about $68.9 \mathrm{eV}$ shown for different effective NIR intensities (i.e. different temporal overlap). The experimental values are shown in the upper panel. Lower panel displays the corresponding simulated angular distribution.

sidebands of the same order might be explained by the additional phase acquired by the electron in the NIR field which is determined by its velocity or wave vector $k$ (see expression (3) for the Volkov phase in paper [9]). On the other hand, the sidebands are situated on both sides of the central line symmetrically in energy $E_{S B}=E_{0} \pm n \hbar \omega_{N I R}$. This leads to non-symmetrical values of $k=k_{0} \pm \delta k$ and thus to a non-symmetrical Volkov phase which finally determines the intensity of the sidebands.

The number of sidebands decreases with increase of the emission angle and turns to zero at $90^{\circ}$ (only the central line remains) in agreement with the experimental results. In addition, in both experiment and theory, the angular distribution of the high order sidebands is concentrated at zero degrees i.e. along the polarization vector of both pulses.

The oscillatory behavior of the central line at higher NIR intensities (see Fig. 3) can be, at least qualitatively, explained by our model. At low NIR intensity, when single photon ionization dominates, the angular distribution has the well known dipole character. With higher NIR intensity (i.e. better overlap of the pulses) an increasing number of NIR quanta are additionally absorbed and emitted, each quantum bringing 
in a unit of angular momentum. For the electron to end up in the central line, it has to absorb and emit the same number of NIR photons. Thus, for the photoionization from the Ne $2 \mathrm{p}(\ell=1)$ shell, having a maximum angular momentum of $\ell=2$ in XUV photoionization, the electron in the central line will progressively get maximum angular momenta of $\ell=4, \ell=6$, etc. due to the absorption and emission of more and more NIR photons as the NIR intensity is increased. The oscillatory nature (additional maxima) in the angular distribution can thus be explained by the interference between the partial waves with different $\ell$.

The strong maximum at $90^{\circ}$ for the central line, at maximum NIR-intensity, is also reproduced in the theoretical model (Fig. 3). Qualitatively this feature can be explained by the fact that the NIR field, acting on the electrons emitted from the atom at $90^{\circ}$, practically does not change their direction of motion and energy for our experimental conditions. In contrast, the electron yield in the central line emitted at small angles around the FEL polarization axis is substantially depleted and redistributed between sidebands as the intensity of the NIR is increased, resulting in an angular distribution that peaks perpendicular to the laser polarization direction. This maximum at $90^{\circ}$ remains the dominant feature also for much higher dressing fields. We note that although there is qualitative agreement between the experimental and theoretical angular distributions in Fig. 3, there are nevertheless some discrepancies, which can be explained by the roughness of the modeling, which ignores the variation of the XUV pulse structure from shot to shot, and by the relatively large error that can result from the sorting procedure. Further experiments, including more statistics and better timeresolution should lead to a better agreement.

A quite different angular distribution pattern is observed for the sidebands. In Fig. 4, the experimental (upper panel) and simulated (lower panel) angular distribution of the second sideband (electron energy $\sim 71.9 \mathrm{eV}$ ) on the high-energy side of the central line is shown for different NIR intensities. The modulation due to multi-photon character of photo absorption is again clearly present. But in contrast to the central line, the angular distribution of the sideband is characterized by a deep minimum at $90^{\circ}$. The theoretical simulation agrees qualitatively with the experiment and reproduces the minimum and the modulation. The minimum at $90^{\circ}$ is again easily explained by the fact that electrons emitted perpendicular to the NIR field polarization undergo a momentum shift under the influence of the NIR field that is perpendicular to their original momentum, which does not change the absolute magnitude of the momentum very much, i.e. does not contribute to the formation of sidebands.

For the discussion of experiments using a SASE (self-amplification of spontaneous emission) FEL the low temporal coherence has to be taken into account. As already experimentally demonstrated in molecular dissociation experiments e.g. [37] the chaotic sub-pulse structure of a SASE FEL pulse shows different experimental signatures as expected from a fully coherent XUV pulse. The sub-pulse width (corresponding to the coherence time) was measured at FLASH by autocorrelation measurements to be within $\sim 1.6$ fs and $\sim 8$ fs depending on the XUV wavelength [38, 39]. For the parameters of 

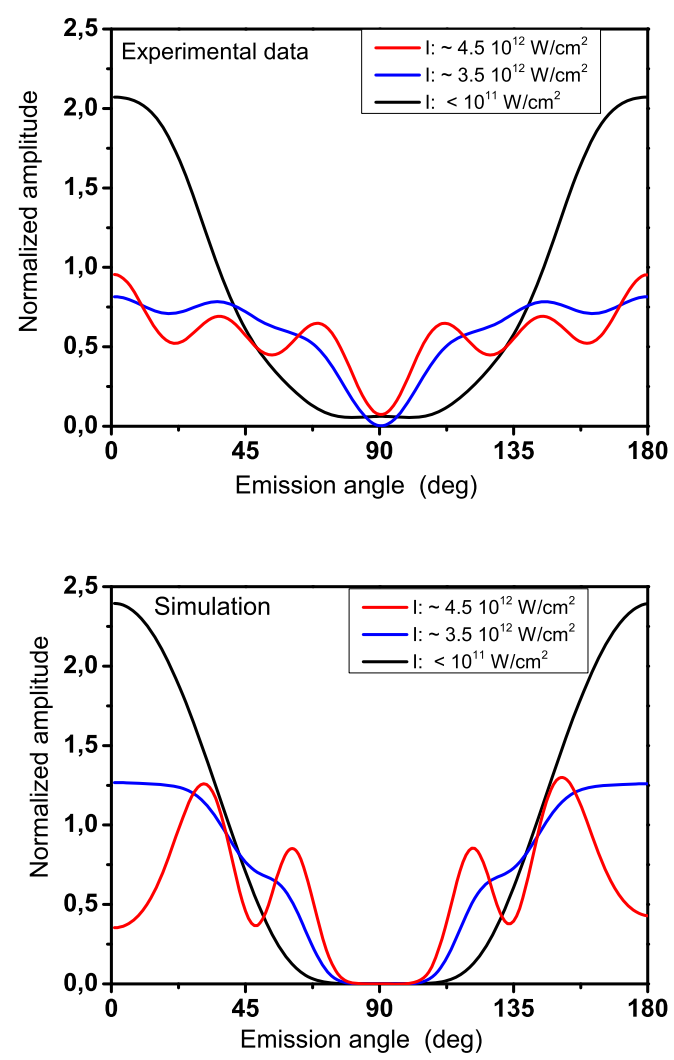

Figure 4. (color online). Angular dependence of the (high energy side) second sideband (electron energy $\sim 71.9 \mathrm{eV}$ ) shown for different effective optical intensities (i.e. different delay times). The experimental results are shown in the upper panel. The lower panel displays the corresponding simulated angular distribution. For better comparison the curves are normalized on the total amount of signal for the sideband.

the current experiment a coherence time of about $3 \mathrm{fs}$ is expected. As shown in Ref. [40] the sideband spectrum is also rather sensitive to the degree of temporal coherence of the XUV pulse. Smaller coherence time leads to an increase of the width of each sideband. The acquired sideband spectra are in good agreement with the expectation and support a coherence time $>1.5$ fs.

In conclusion, we have investigated the angular distribution of photoelectrons from Ne atoms created by XUV FEL radiation in the presence of a strong NIR dressing field using velocity-map-imaging spectroscopy. Extended sideband structures with clear signatures of interferences in the energy spectra and in the angular distributions were observed, showing evidence of a gross structure. The measured oscillatory structure of the angular distributions of the sidebands as well as the central line are dominated by multi-photon processes in the NIR dressing field, which give rise to the formation of electrons with high angular momenta resulting in the oscillatory structures. Even averaging over a range of NIR intensities these structures are still present and demonstrate the universal character of the interference effects. The experimental 
results are qualitatively explained by the theoretical model based on the strong field approximation.

\section{Acknowledgments}

We want to acknowledge the work of the scientific and technical team at FLASH, in particular N. Gerasimova for the analysis of the FEL pulse duration by spectral correlations. N.M.K. is grateful to European XFEL for hospitality and financial support. L.R. and P.J. acknowledge the support from the Swedish Research Council and the Swedish Foundation for Strategic Research.

\section{References}

[1] Ackermann W et al. 2007 Nature Photonics 1336

[2] Emma P et al. 2010 Nature Photonics 4641

[3] Ishikawa T et al. 2012 Nature Photonics 6540

[4] Sorokin A et al. 2007 Phys. Rev. Lett. 99213002

[5] Young L et al. 2010 Nature 46656

[6] Rohringer $\mathrm{N}$ et al. 2012 Nature $\mathbf{4 8 1} 488$

[7] Mazza T et al. 2012 J. Phys. B: At. Mol. Opt. Phys. 45141001

[8] Krikunova M et al. 2011 J. Chem. Phys. 134024313

[9] Kazansky A K, Sazhina I P and Kabachnik N M 2010 Phys. Rev. A 82033420

[10] Schins J M et al. 1994 Phys. Rev. Lett. 732180 and 1995 Phys. Rev. A 521272

[11] Mauritsson J et al. 2004 Phys. Rev. A 70 021801(R)

[12] Paul P M et al. 2001 Science 2921689

[13] Meyer M et al. 2008 Phys. Rev. Lett. 101193002

[14] P. Radcliffe, et al. 2012 New J. Phys. 14043008

[15] Haber L H , Doughty B and Leone S R 2011 Phys. Rev. A 84013416 and 2009 J. Phys. Chem. A 11313152

[16] Aseyev S A 2003 Phys. Rev. Lett. 91223902

[17] Rudenko A et al. 2004 J. Phys. B: At. Mol. Opt. Phys. 37 L407

[18] Morishita T et al. 2007 Phys. Rev. A 75023407

[19] Kumarappan V et al. 2008 Phys. Rev. Lett. 100093006

[20] Marchenko T et al. 2010 J. Phys. B 43185001

[21] Arbo D G 2010 Phys. Rev. A 81 021403(R) and 201082043426

[22] Arbo D G 2011 Nucl. Instr. Meth. B 27924

[23] Kazansky A K and Kabachnik N M 2009 J. Phys. B 42121002

[24] Kazansky A K, Sazhina I P and Kabachnik N M 2009 J. Phys. B 42245601

[25] Kazansky A K and Kabachnik N M 2010 J. Phys. B 43035601

[26] Meyer M et al. 2012 Phys. Rev. Lett. 108063007

[27] Redlin H et al. 2011 Nucl. Instr. Meth. A 63588

[28] Eppink A T J B et al. 1997 Appl. Opt. 311146

[29] Vrakking M J J 2001 Rev. Sci. Instr. 724084

[30] Johnsson P et al. 2010 Optics Letters 354163

[31] Agostini P et al. 1979 Phys. Rev. Lett. 421127

[32] Bauch S and Bonitz M 2008 Phys. Rev. A 78043403

[33] Volkov D B 1935 Z. Phys 94250

[34] Kazansky A K and Kabachnik N M 2007 J. Phys. B 402163 
Interference in the angular distribution of photoelectrons in superimposed XUV and optical laser fields11

[35] Herman F and Skillman S 1963 Atomic Structure Calculations (Prentice-Hall, Englewood Cliffs, NJ)

[36] Kennedy D J and Manson S T 1972 Phys. Rev. A 5227

[37] Jiang Y H et al. 2010 Phys. Rev. A 82 041403(R)

[38] Roling S et al. 2011 Phys. Rev. ST Accel. Beams 14080701

[39] Schlotter W F et al. 2010 Opt. Lett. 35372

[40] Kazansky A K , Sazhina I P and Kabachnik N M 2012 Phys. Rev. A 86033404 